\title{
Hydrogen sulfide protects H9c2 cardiac cells against doxorubicin-induced cytotoxicity through the PI3K/Akt/FoxO3a pathway
}

\author{
MI-HUA LIU ${ }^{1 *}$, YUAN ZHANG $^{2 *}$, JUN HE$^{1 *}$, TIAN-PING TAN ${ }^{1}$, SHAO-JIAN WU ${ }^{1}$, \\ DONG-MING GUO ${ }^{3}$, HUI HE ${ }^{4}$, JUAN PENG ${ }^{4}$, ZHI-HAN TANG ${ }^{4}$ and ZHI-SHENG JIANG ${ }^{4}$
}

\author{
${ }^{1}$ Department of Clinical Laboratory, Affiliated Nanhua Hospital, University of South China, Hengyang, Hunan 421001; \\ ${ }^{2}$ Department of Pathology, Mawangdui Hospital, Changsha, Hunan 410016; ${ }^{3}$ Laboratory of Clinical Anatomy, \\ University of South China, Hengyang, Hunan $421001 ;{ }^{4}$ Institute of Cardiovascular Disease, Key Laboratory for \\ Arteriosclerology of Hunan Province, University of South China, Hengyang, Hunan 421001, P.R. China
}

Received February 12, 2015; Accepted March 30, 2016

DOI: $10.3892 /$ ijmm.2016.2563

\begin{abstract}
Doxorubicin (DOX) is an efficient drug used in cancer therapy that also produces reactive oxygen species (ROS) that induces severe cytotoxicity, which limits its clinical application. Hydrogen sulfide $\left(\mathrm{H}_{2} \mathrm{~S}\right)$, a novel gasotransmitter, has been shown to exert cardioprotective effects. The present study aimed to determine whether exogenous $\mathrm{H}_{2} \mathrm{~S}$ protects $\mathrm{H} 9 \mathrm{c} 2$ cardiac cells against DOX-induced cytotoxicity and whether these protective effects are mediated through the PI3K/Akt/FoxO3a pathway. The $\mathrm{H} 9 \mathrm{c} 2$ cardiac cells were exposed to $5 \mu \mathrm{M}$ DOX for $24 \mathrm{~h}$ to establish a model of DOX-induced cardiotoxicity. The results showed that the treatment of H9c2 cardiac cells with sodium hydrosulfide (NaHS) for 30 min prior to DOX exposure markedly attenuated the phosphorylation of Akt and FoxO3a. Notably, pre-treatment of the H9c2 cells with NaHS significantly attenuated the nuclear localization of FoxO3a as well as the apoptosis of $\mathrm{H} 9 \mathrm{c} 2$ cells induced by DOX. The treatment of H9c2 cells with N-acetyl-L-cysteine (NAC), a scavenger of ROS, prior to DOX exposure, also markedly increased the phosphorylation of Akt and FoxO3a which was inhibited by DOX alone. Furthermore, pre-treatment with LY294002, a selective inhibitor of PI3K/Akt, reversed the protective effect
\end{abstract}

Correspondence to: Dr Mi-Hua Liu, Department of Clinical Laboratory, Affiliated Nanhua Hospital, University of South China, 336 Dongfeng South Road, Hengyang, Hunan 421001, P.R. China

E-mail: mihualiu@163.com

Dr Zhi-Sheng Jiang, Institute of Cardiovascular Disease, Key Laboratory for Arteriosclerology of Hunan Province, University of South China, 28 West Changsheng Road, Hengyang, Hunan 421001, P.R. China

E-mail: zsjianglab@aliyun.com

${ }^{*}$ Contributed equally

Key words: hydrogen sulfide, doxorubicin, FoxO3a, apoptosis, cardiomyocytes of $\mathrm{H}_{2} \mathrm{~S}$ against DOX-induced injury of cardiomyocytes, as demonstrated by an increased number of apoptotic cells, a decrease in cell viability and the reduced phosphorylation of Akt and FoxO3a. These findings suggested that exogenous $\mathrm{H}_{2} \mathrm{~S}$ attenuates DOX-induced cytotoxic effects in $\mathrm{H} 9 \mathrm{c} 2$ cardiac cells through the PI3K/Akt/FoxO3a pathway.

\section{Introduction}

Doxorubicin (DOX) is one of the most widely used anticancer drugs because of its potent therapeutic effects on various types of cancer, including leukemia, lymphoma and breast cancer. However, the clinical use of DOX is limited by severe cardiotoxicity, which may lead to dilated cardiomyopathy and congestive heart failure (1). The production of reactive oxygen species (ROS) is involved in the toxic effect elicited by DOX on cardiomyocytes and endothelial cells, and in the promotion of endothelial dysfunction (2) and apoptosis (3). A number of pharmacological interventions have been suggested as therapies to protect against DOX-induced cardiotoxicity.

Hydrogen sulfide $\left(\mathrm{H}_{2} \mathrm{~S}\right)$ is considered a toxic gas, and has been classified as the third gasotransmitter, together with nitric oxide and carbon monoxide, and exerts various effects on the cardiovascular system (4). Previous findings have shown that $\mathrm{H}_{2} \mathrm{~S}$ protects the heart from myocardial ischemia-reperfusion (IR) injury (5). In a previous study, it was demonstrated that the increased generation of endogenous $\mathrm{H}_{2} \mathrm{~S}$ in the early reperfusion phase has an important function in ischemic pre-conditioning-elicited protection in isolated hearts (6).

The forkhead box class $\mathrm{O}$ (FoxO) subfamily of forkhead transcription factors comprises the members FoxO1, FoxO3a and FoxO4, which are downstream targets of Akt (7). FoxO3a is involved in the regulation of various cell processes, including proliferation and apoptosis, as well as protection against oxidative stress and metabolism (8). A model of $\beta$-amyloid-induced neuron death was used to demonstrate that FoxO3a is activated, translocates to the nucleus, and subsequently mediates neuron death through Bim (9). In neonatal rat ventricular myocytes (NRVMs), hyperglycemia was demonstrated to 
markedly enhance the apoptosis of NRVMs through the translocation of FoxO3a to the nucleus, and the resultant enhanced transcriptional activity of FoxO3a (10). Li et al found that the $\mathrm{PI} 3 \mathrm{~K} / \mathrm{Akt} /$ FoxO3a pathway is involved in neuronal apoptosis in the brain of a developing rat (11). In a previous study, it was demonstrated that resveratrol protects $\mathrm{PC} 12$ cells against high glucose-induced oxidative stress and apoptosis through the activation of the PI3K/Akt/FoxO3a signaling pathway (12). In addition, sodium tanshinone IIA sulfonate (13) and bromelain (14) have been found to protect rat hearts against IR injury through the activation of the PI3K/Akt/FoxO3a pathway. Therefore, we hypothesized that the PI3K/Akt/FoxO3a pathway may be involved in the protective effect of exogenous $\mathrm{H}_{2} \mathrm{~S}$ against DOX-induced cardiotoxicity in $\mathrm{H} 9 \mathrm{c} 2$ cardiac cells.

To examine this hypothesis, H9c2 cells were treated with $5 \mu \mathrm{M}$ DOX in the present study to establish a model of chemotherapy-induced cardiotoxicity as previously described (15). Subsequently, we examined: i) the effect of DOX on the phosphorylation of Akt and FoxO3a; ii) the effect of exogenous $\mathrm{H}_{2} \mathrm{~S}$ on the DOX-induced translocation of FoxO3a to the nucleus where it subsequently mediates $\mathrm{H} 9 \mathrm{c} 2$ cardiac cell death through Bim; and iii) whether exogenous $\mathrm{H}_{2} \mathrm{~S}$ protects $\mathrm{H} 9 \mathrm{c} 2$ cells against DOX-induced cardiotoxicity through the $\mathrm{PI} 3 \mathrm{~K} / \mathrm{Akt} / \mathrm{FoxO} 3 \mathrm{a}$ pathway.

\section{Materials and methods}

Materials. Methyl thiazolyl tetrazolium (MTT), Hoechst 33258 , 2',7'-dichlorofluorescein diacetate (DCFH-DA), DOX, sodium hydrosulfide (NaHS), and N-acetyl-L-cysteine (NAC) were purchased from Sigma-Aldrich (St. Louis, MO, USA). LY294002 was purchased from Calbiochem (Billerica, MA, USA). Cell culture medium components were purchased from Thermo Fisher Scientific (Waltham, MA, USA) unless otherwise noted. The H9c2 cardiac myocytes were obtained from the Shanghai Cell Library of China [originally from the American Type Culture Collection (ATCC); Manassas, VA, USA].

Cell culture. The H9c2 cardiac cells were cultured in Dulbecco's modified Eagle's medium (DMEM) supplemented with $10 \%$ fetal bovine serum (FBS), $100 \mu \mathrm{g} / \mathrm{ml}$ streptomycin (Life Technologies, Carlsbad, CA, USA) and $100 \mathrm{U} / \mathrm{ml}$ penicillin-streptomycin (Life Technologies) in a humidified $5 \% \mathrm{CO}_{2}$ atmosphere at $37^{\circ} \mathrm{C}$. The $\mathrm{H} 9 \mathrm{c} 2$ cardiac myocytes were passaged every 2 days Subsequently, they were seeded at a density of $2 \times 10^{6}$ cells/dish in $100-\mathrm{mm}$ dishes with $10 \%$ calf serum, incubated for $24 \mathrm{~h}$ and then, the medium was replaced with $0.5 \%$ FBS DMEM for 24-h serum starvation. To determine the degree of apoptosis, the H9c2 cardiac myocytes were treated with NaHS $(100 \mu \mathrm{M})$ for $30 \mathrm{~min}$ or NAC $(1,000 \mu \mathrm{M})$ for $60 \mathrm{~min}$, followed by exposure to DOX for $24 \mathrm{~h}$. In some experiments, the H9c2 cells were treated with LY294002 (50 $\mu \mathrm{M})$ prior to NaHS stimulation.

MTT assay. The MTT assay is a standard method used to assess cell viability.Prior to each experiment, the $\mathrm{H} 9 \mathrm{c} 2$ cardiac myocytes $\left(5 \times 10^{2}\right.$ cells/well) were seeded in 96-well microtiter plates. After incubation with the phosphatidylinositol-3-kinase (PI3K) inhibitor LY294002 (50 $\mu \mathrm{M})$ and/or NaHS for $30 \mathrm{~min}$, the cells were exposed to $5 \mu \mathrm{M}$ DOX for a further $24 \mathrm{~h}$. Subsequently, $10 \mu \mathrm{l}$ MTT solution was added to each well, and the plates were incubated for $4 \mathrm{~h}$ at $37^{\circ} \mathrm{C}$. The absorbance was measured at $470 \mathrm{~nm}$ using a SpectraMax 190 Absorbance Microplate Reader (Molecular Devices LLC, Sunnyvale, CA, USA) and used to calculate the relative ratio of cell viability. Three independent experiments were performed for each experimental condition.

Assessment of H9c2 cell apoptosis. The analysis of apoptosis was performed by fluorescence microscopy with the chromatin dye Hoechst 33258. After various treatments, the cells were fixed in ice-cold $4 \%$ paraformaldehyde dissolved in phosphate-buffered saline (PBS) at room temperature for $20 \mathrm{~min}$. Non-specific binding was blocked using $5 \%$ normal goat serum in $0.01 \mathrm{M}$ PBS containing $0.3 \%$ Triton X-100 (PBS+T). The cells were washed twice with PBS and incubated with $10 \mu \mathrm{g} / \mathrm{ml}$ Hoechst 33258 for $15 \mathrm{~min}$ at room temperature in the dark. The cells were visualized under a fluorescence microscope (BX50-FLA; Olympus, Tokyo, Japan). Apoptotic cells exhibited condensed, fractured or distorted nuclei, whereas viable cells exhibited normal nuclear size and uniform fluorescence.

Measurement of intracellular ROS levels. The determination of intracellular ROS levels was performed by measuring the level of a fluorescent product formed by the oxidation of DCFH-DA. Briefly, the culture medium was plated into 96-well microtiter plates. After various treatments, the cells were washed with PBS 3 times. Following the addition of fresh culture medium, the cells were incubated with DCFH-DA at a final concentration of $10 \mu \mathrm{mol} / 1$ for $30 \mathrm{~min}$ at $37^{\circ} \mathrm{C}$. The cells were washed again with PBS 3 times and then lysed with $500 \mu 1$ 90\% DMSO and $10 \%$ PBS for $10 \mathrm{~min}$ at room temperature in the dark. The supernatant $(200 \mu \mathrm{l})$ was transferred to another 96-well microtiter plate. The fluorescence intensity of the oxidized product, 2',7'-dichlorofluorescein (DCF), was measured using a Model F-4500 fluorescence spectrophotometer (Hitachi, Tokyo, Japan) at $495 \mathrm{~nm}$ (excitation) and $520 \mathrm{~nm}$ (emission). The values were expressed as the percentage of fluorescence intensity relative to the control wells.

Western blot analysis. H9c2 cells were treated with 0.1, 1, 5 and $10 \mu \mathrm{M}$ DOX for $24 \mathrm{~h}$ (Fig. 1) and exposed to $5 \mu \mathrm{M}$ DOX for 3, 6, 12 and $24 \mathrm{~h}$ (Fig. 2). The cells were homogenized directly in cell lysis buffer (Cell Signaling Technology, Danvers, MA, USA) and phosphatase inhibitor cocktail (Sigma-Aldrich). The lysates were centrifuged at $12000 \mathrm{x}$ g for $10 \mathrm{~min}$ at $4^{\circ} \mathrm{C}$. The protein concentration was determined with the use of a BCA protein assay kit according to the manufacturer's instructions. For nuclear/cytoplasmic fractionation, the cultured $\mathrm{H} 9 \mathrm{c} 2$ cells were fractionated into nuclear and cytoplasmic lysates using a Nuclear and Cytoplasmic Protein Extraction kit (Beyotime Institute of Biotechnology, Shanghai, China) according to the manufacturer's instructions. The extracted proteins were mixed with 5\% sodium dodecyl sulfate (SDS)-PAGE sample buffer, boiled at $100^{\circ} \mathrm{C}$ for $7 \mathrm{~min}$ and then separated by electrophoresis on a $10 \%$ SDS-polyacrylamide gel. Following electrophoresis, the proteins were transferred to polyvinylidene difluoride membranes (Beyotime Institute of Biotechnology). The membranes were blocked in Tris-buffered saline (TBS) containing $0.1 \%$ Tween-20 (TBS-T) with 5\% non-fat dry milk, for $2 \mathrm{~h}$ at room temperature, with rotation. After blocking, the membranes were incubated with the following antibodies: rabbit anti-Akt polyclonal antibody (cat. no. 9272, 1:2,000), rabbit anti-phosphorylated (p-) 
A
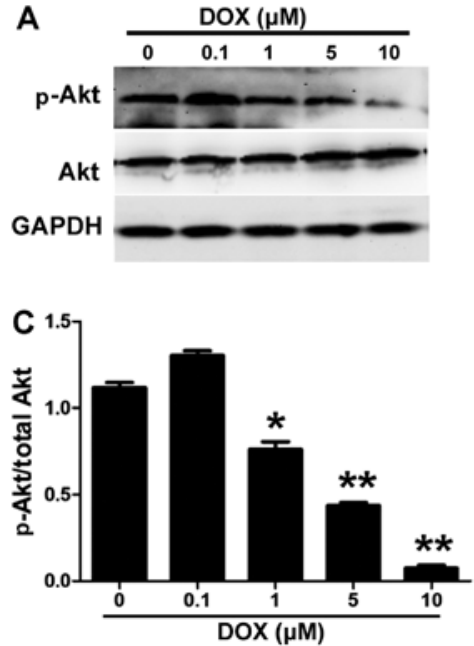

B
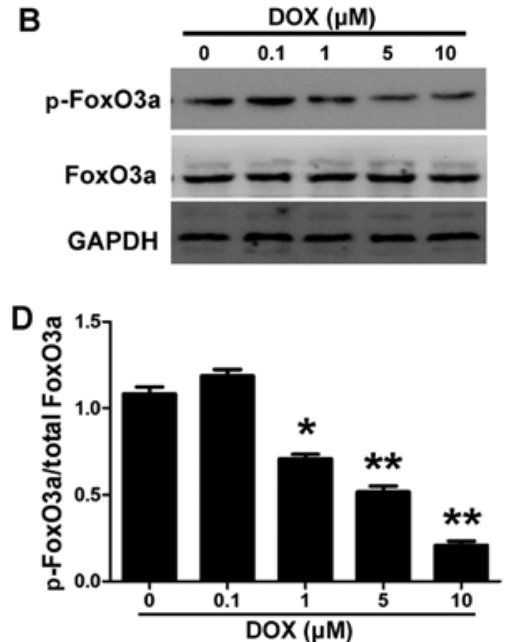

Figure 1. Doxorubicin (DOX) decreases the expression of phosphorylated (p)-Akt and p-FoxO3a in H9c2 cells in a dose-dependent manner. H9c2 cells were exposed to DOX at different concentrations for $24 \mathrm{~h}$. (A and B) Phosphorylation of Akt and FoxO3a was determined using western blot analysis (C and D) Relative levels of p-FoxO3a versus total FoxO3a, and p-Akt versus total Akt in each sample as determined by blot densitometry. Data are shown as the means $\pm \operatorname{SEM}(\mathrm{n}=3) .{ }^{*} \mathrm{P}<0.05,{ }^{* *} \mathrm{P}<0.01$ compared with the control group.
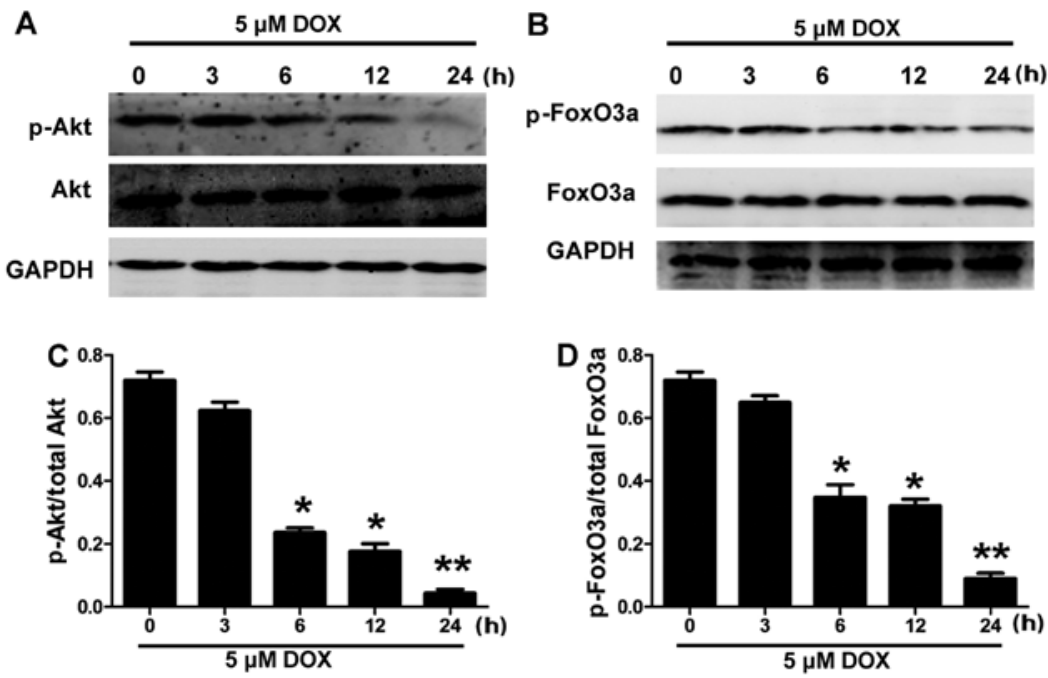

Figure 2. Doxorubicin (DOX) decreases the expression of phosphorylated (p)-Akt and p-FoxO3a in H9c2 cells in a time-dependent manner. H9c2 cells were exposed to $5 \mu \mathrm{M}$ DOX for the indicated time periods. (A and B) Phosphorylation of Akt and FoxO3a was determined using western blot analysis. (C and D) Relative levels of p-FoxO3a versus total FoxO3a, and p-Akt versus total Akt in each sample as determined by blot densitometry. Data are shown as the means \pm SEM $(\mathrm{n}=3)$. ${ }^{*} \mathrm{P}<0.05,{ }^{* *} \mathrm{P}<0.01$ compared with the control group.

Akt (Ser 473) monoclonal antibody (cat. no. 4060, 1:2,000), rabbit anti-FoxO3a polyclonal antibody (cat. no. 12829, 1:2,000), rabbit anti-p-FoxO3a (ser 253) polyclonal antibody (cat. no. 9466, 1:1,000) (all from Cell Signaling Technology) and rabbit anti-Bim polyclonal antibody (ab32158, 1:200; Abcam, Cambridge, MA, USA). The membranes were then incubated in $5 \%$ milk or bovine serum albumin overnight at $4^{\circ} \mathrm{C}$. The primary antibody was removed by washing the membranes 3 times in TBS-T, and subsequently incubating the membranes for $2 \mathrm{~h}$ with the appropriate horseradish peroxidase-conjugated secondary antibodies. After washing the membranes 3 times in TBS-T, the antigen-antibody bands were detected using an enhanced chemiluminescence reagent kit and quantified using a densitometry program. The data from the western blot analysis of p-Akt and p-FoxO3a were presented as a ratio of the $\mathrm{p}$-forms to their total forms, respectively. The immunoblot of Bim was corrected to the bands of GAPDH.
Statistical analysis. The results are presented as the means \pm SEM. Statistical analysis was performed using the Student's t-test or analysis of variance (ANOVA) with SPSS 13.0 software (SPSS, Inc., Chicago, IL, USA). In all cases, $\mathrm{P}<0.05$ was considered to indicate a statistically significant difference.

\section{Results}

DOX decreases the phosphorylation of Akt and FoxO3a in $\mathrm{H} 9 \mathrm{c} 2$ cells. To investigate the role of the PI3K/Akt/FoxO3a pathway in DOX-induced cytotoxicity, we investigated the phosphorylation of Akt and FoxO3a in the H9c2 cells following exposure to DOX. The H9c2 cells were treated with DOX at different concentrations for different time periods, and the effect on the phosphorylation of Akt and FoxO3a was determined using western blot analysis. Figs. 1 and 2 show that DOX decreased 

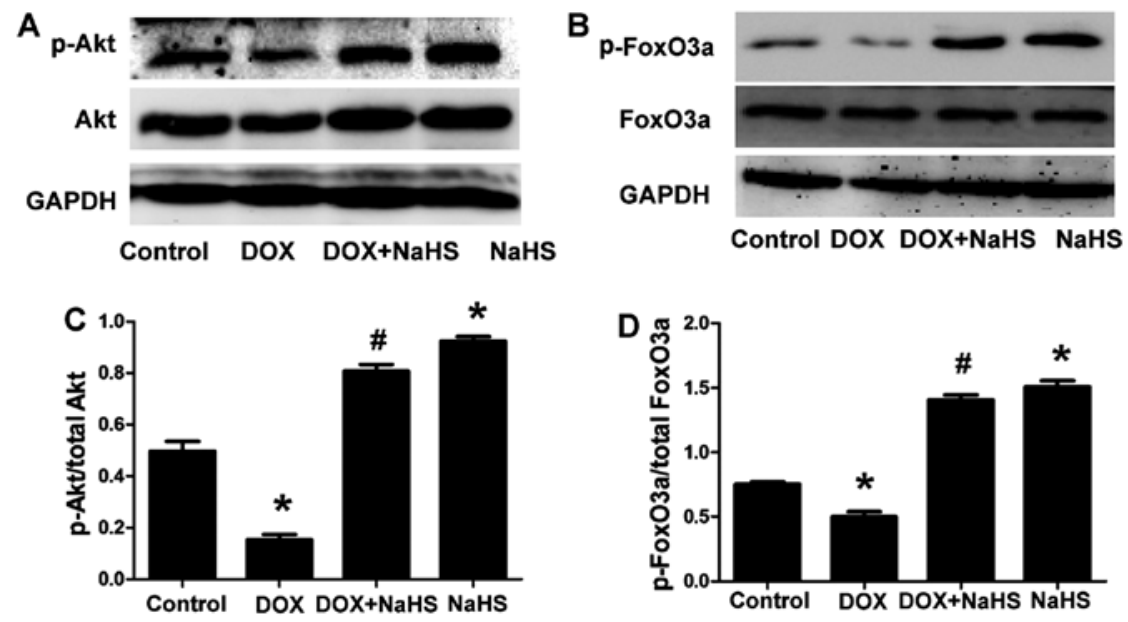

Figure 3. Effect of hydrogen sulfide $\left(\mathrm{H}_{2} \mathrm{~S}\right)$ on the doxorubicin (DOX)-induced decrease in the expression of phosphorylated (p)-Akt and p-FoxO3a in H9c2 cells. H9c2 cells were exposed to $5 \mu \mathrm{M}$ DOX for $24 \mathrm{~h}$ and then cells were treated with $100 \mu \mathrm{M}$ sodium hydrosulfide (NaHS) for $30 \mathrm{~min}$ prior to DOX exposure or untreated. (A and B) Phosphorylation of Akt and FoxO3a was examined using western blot analysis. (C and D) Relative levels of p-FoxO3a versus total FoxO3a and p-Akt versus total Akt in each sample as determined by blot densitometry. Data are shown as the means $\pm \mathrm{SEM}(\mathrm{n}=3)$. ${ }^{*} \mathrm{P}<0.05$, compared with the control group; ${ }^{\#} \mathrm{P}<0.05$, compared with the DOX-treated group.
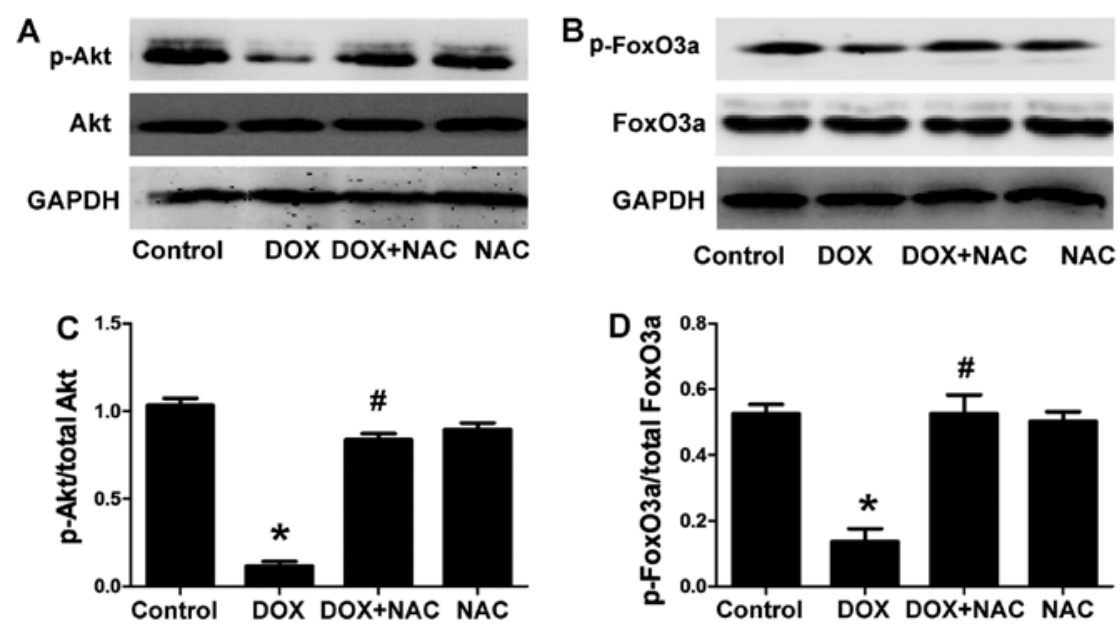

Figure 4. Effect of N-acetyl-L-cysteine (NAC) on the doxorubicin (DOX)-induced decrease in the expression of phosphorylated (p)-Akt and p-FoxO3a in H9c2 cells. H9c2 cells were exposed to $5 \mu \mathrm{M}$ DOX for $24 \mathrm{~h}$ and then the cells were either treated with 1,000 $\mu \mathrm{M}$ NAC for 60 min or untreated. (A and B) Western blot analysis was applied to detect changes in the phosphorylation of Akt and FoxO3a. (C and D) Relative levels of p-FoxO3a versus total FoxO3a, and p-Akt versus total Akt in each sample as determined by blot densitometry. Data are shown as the means $\pm \mathrm{SEM}(\mathrm{n}=3)$. ${ }^{*}<0.05$, compared with the control group; ${ }^{\text {"}} \mathrm{P}<0.05$, compared with the DOX-treated group.

the phosphorylation of Akt and FoxO3a in the H9c2 cells in a concentration- and time-dependent manner. DOX inhibited the phosphorylation of Akt and FoxO3a in the H9c2 cells at a concentration of $1 \mu \mathrm{M}$ and the maximal effect was reached at a concentration of $10 \mu \mathrm{M}$ (Fig. 1). Fig. 2 shows that $5 \mu \mathrm{M}$ DOX induced a significant decrease in the levels of p-Akt and p-FoxO3a at 6 and $12 \mathrm{~h}$, and it almost completely abolished the phosphorylation of Akt and FoxO3a at $24 \mathrm{~h}$ in the $\mathrm{H} 9 \mathrm{c} 2$ cells.

Exogenous $\mathrm{H}_{2} \mathrm{~S}$ ameliorates the DOX-induced decrease in the levels of $p$-Akt and p-FoxO3a in H9c2 cells. To determine whether the cytoprotective effect of $\mathrm{H}_{2} \mathrm{~S}$ against DOX-induced toxicity was associated with the PI3K/Akt/FoxO3a pathway in $\mathrm{H} 9 \mathrm{c} 2$ cells, we examined the effect of $\mathrm{NaHS}$ on the expression of p-Akt and p-FoxO3a. The results showed that treating the H9c2 cells with $100 \mu \mathrm{M}$ NaHS (a donor of $\mathrm{H}_{2} \mathrm{~S}$ ) for $30 \mathrm{~min}$ prior to exposure to $5 \mu \mathrm{M}$ DOX for $24 \mathrm{~h}$ significantly increased the phosphorylation of Akt and FoxO3a (Fig. 3). Furthermore, NaHS treatment alone also significantly increased the levels of p-Akt and p-FoxO3a compared with the DOX-treated groups. The total Akt and FoxO3a levels remained unchanged among the four groups. These results suggested that the PI3K/Akt/FoxO3a pathway was involved in the protective effect of $\mathrm{H}_{2} \mathrm{~S}$.

NAC ameliorates the DOX-induced decrease in the levels of $p$ - $A k t$ and p-FoxO3a in H9c2 cells. The H9c2 cells were treated with $1,000 \mu \mathrm{M}$ NAC (ROS scavenger) for $60 \mathrm{~min}$ prior to exposure to $5 \mu \mathrm{M}$ DOX for $24 \mathrm{~h}$, to confirm whether the protective effect of $\mathrm{H}_{2} \mathrm{~S}$ on the DOX-induced decrease in p-Akt and p-FoxO3a is associated with antioxidation. As shown in Fig. 4, the pretreatment of the $\mathrm{H} 9 \mathrm{c} 2$ cells with NAC markedly increased the expression of $\mathrm{p}$-Akt and $\mathrm{p}$-FoxO3a and this was similar to the protective effect observed with NaHS pre-treatment. The total Akt and FoxO3a levels remained unchanged in the four groups. 

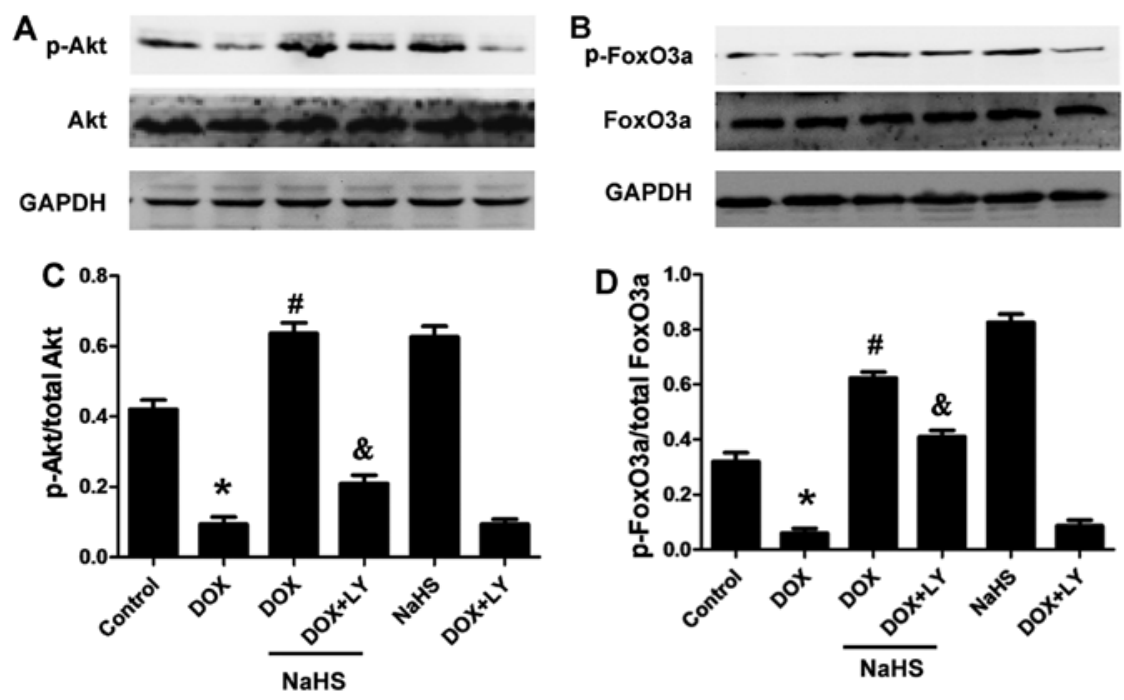

Figure 5. LY294002 inhibits the effect of hydrogen sulphide $\left(\mathrm{H}_{2} \mathrm{~S}\right)$ on Akt and FoxO3a phosphorylation in $\mathrm{H} 9 \mathrm{c} 2$ cells. H9c2 cells were exposed to $5 \mu \mathrm{M}$ doxorubicin (DOX) for $24 \mathrm{~h}$ and were untreated or treated with $100 \mu \mathrm{M}$ sodium hydrosulfide (NaHS) and/or $50 \mu \mathrm{M} \mathrm{LY} 294002$ for 30 min prior to DOX exposure. (A and B) Phosphorylation of Akt and FoxO3a was determined using western blot analysis. (C and D) Relative levels of p-FoxO3a versus total FoxO3a, and p-Akt versus total Akt in each sample as determined by blot densitometry. Data are shown as the means $\pm \mathrm{SEM}(\mathrm{n}=3)$. ${ }^{*} \mathrm{P}<0.05$, compared with the control group; ${ }^{~} \mathrm{P}<0.05$, compared with the DOX-treated group; ${ }^{\&} \mathrm{P}<0.05$, compared with the NaHS+DOX group. LY, LY294002.
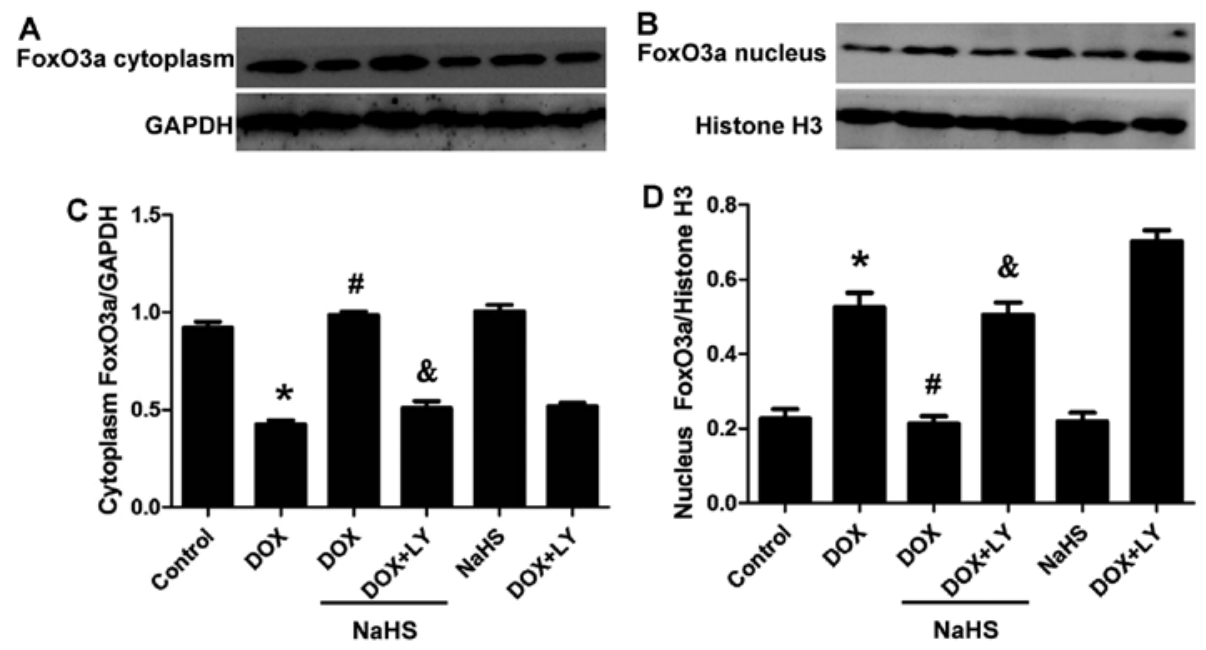

Figure 6. LY294002 inhibits the effect of hydrogen sulphide $\left(\mathrm{H}_{2} \mathrm{~S}\right)$ on FoxO3a nuclear translocation. H9c2 cells were treated with $5 \mu \mathrm{M}$ doxorubicin (DOX) for $24 \mathrm{~h}$ and either untreated or pretreated with $100 \mu \mathrm{M}$ sodium hydrosulfide (NaHS) and/or $50 \mu \mathrm{M} \mathrm{LY} 294002$ for 30 min prior to DOX exposure. (A and B) Protein levels of nuclear and cytosolic FoxO3a were determined using western blot analysis. (C and D) Quantification of the nuclear and cytosolic protein levels of FoxO3a. Data are shown as the means \pm SEM $(n=3)$. "P $<0.05$, compared with the control group; ${ }^{\sharp} \mathrm{P}<0.05$, compared with the DOX-treated group; ${ }^{\&} \mathrm{P}<0.05$, compared with the NaHS+DOX group. LY, LY294002.

The results revealed that an antioxidant effect contributed to the protective effect of $\mathrm{H}_{2} \mathrm{~S}$ against DOX-induced injuries.

Exogenous $\mathrm{H}_{2} \mathrm{~S}$ induces Akt and FoxO3a phosphorylation through-the PI3K/Akt pathway in $\mathrm{H} 9 \mathrm{c} 2$ cells. To investigate the role of the PI3K/Akt pathway in the protective effects of $\mathrm{H}_{2} \mathrm{~S}$, the H9c2 cells were treated with the PI3K inhibitor LY294002 prior to exposure to NaHS plus DOX. The activation of Akt and FoxO3a was determined as described above. LY294002 abolished the stimulation of p-Akt and p-FoxO3a in the presence of NaHS (Fig. 5), but elicited no effect on the expression levels of total Akt and FoxO3a. These results suggested that the $\mathrm{PI} 3 \mathrm{~K} / \mathrm{Akt} / \mathrm{FoxO} 3 \mathrm{a}$ pathway was involved in the protective effect of $\mathrm{H}_{2} \mathrm{~S}$.
DOX enhances the nuclear localization of FoxO3a in $\mathrm{H} 9 \mathrm{c} 2$ cells whereas $\mathrm{H}_{2} \mathrm{~S}$ blocks the effect of DOX. The transcription factor FoxO3a functions through its phosphorylation and subcellular localization. The phosphorylation of FoxO3a by Akt causes it to localize in the cytoplasm and inhibit the functions of FoxO3a, including pro-apoptotic effects (25). By contrast, dephosphorylation of this protein promotes the translocation of FoxO3a to the nucleus and triggers apoptosis. To investigate the effects of $\mathrm{H}_{2} \mathrm{~S}$ and DOX on FoxO3a, we studied the subcellular localization of FoxO3a following exposure to these reagents. Nuclear and cytosolic proteins from $\mathrm{H} 9 \mathrm{c} 2$ cells were extracted, and the subcellular localization of FoxO3a was determined. Fig. 6 shows that DOX enhanced the nuclear localization of FoxO3a in the H9c2 cells, whereas 


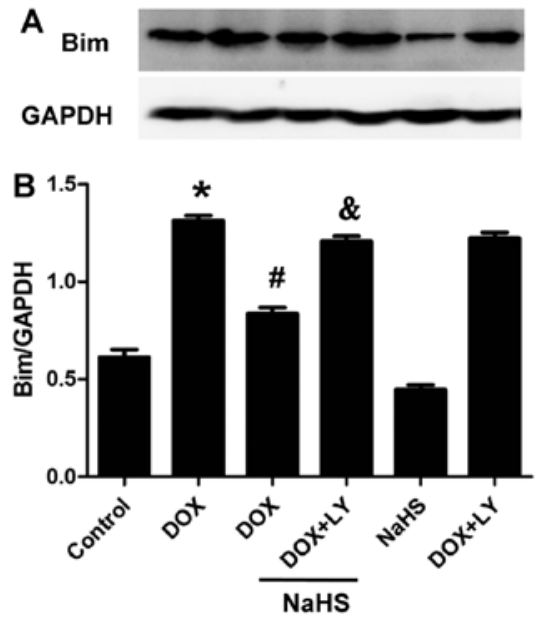

Figure 7. LY294002 inhibits the effect of hydrogen sulfide $\left(\mathrm{H}_{2} \mathrm{~S}\right)$ on doxorubicin (DOX)-induced Bim expression. H9c2 cells were exposed to $5 \mu \mathrm{M}$ DOX for $24 \mathrm{~h}$ and were either untreated or treated with $100 \mu \mathrm{M}$ sodium hydrosulfide (NaHS) and/or $50 \mu \mathrm{M} \mathrm{LY} 294002$ for $30 \mathrm{~min}$ prior to DOX exposure. (A) Bim protein expression was determined using western blot analysis. (B) Relative levels of Bim in each sample as determined by blot densitometry. Data are shown as the means \pm SEM $(n=3)$. "P $<0.05$, compared with the control group; ${ }^{\text {}} \mathrm{P}<0.05$, compared with the DOX-treated group; ${ }^{\&} \mathrm{P}<0.05$, compared with the NaHS + DOX group. LY, LY294002.
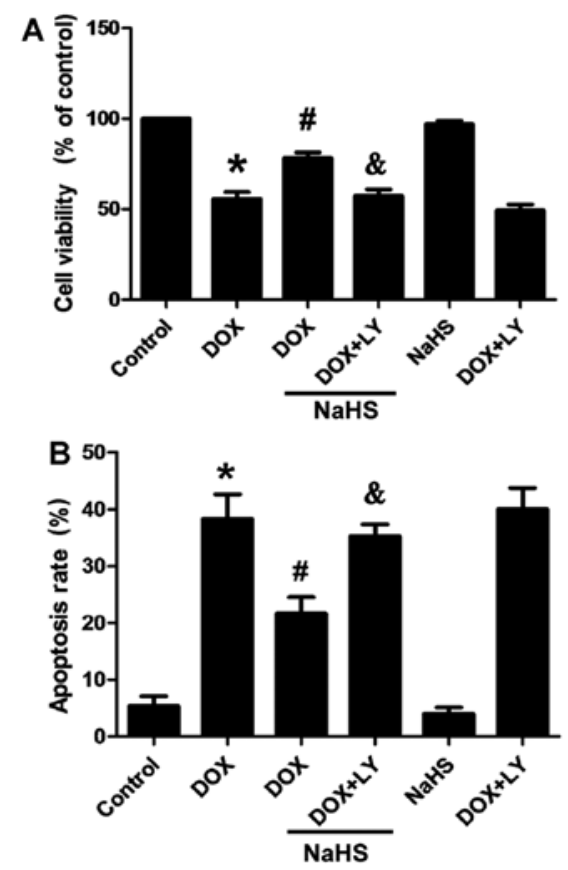

Figure 8. Exogenous hydrogen sulfide $\left(\mathrm{H}_{2} \mathrm{~S}\right)$ protects $\mathrm{H} 9 \mathrm{c} 2$ cells against doxorubicin (DOX)-induced injuries. (A) Cell viability was measured using the MTT assay. (B) Cell apoptosis was measured using Hoechst 33258 nuclear staining. Data are shown as the means \pm SEM $(n=3)$. "P $<0.05$, compared with the control group; ${ }^{~} \mathrm{P}<0.05$, compared with the DOX-treated group; ${ }^{\circledR} \mathrm{P}<0.05$, compared with the sodium hydrosulfide (NaHS) + DOX group. LY, LY294002.

NaHS blocked the effect of DOX. Co-treatment with the PI3K inhibitor, LY294002, abolished the protective effect of NaHS.

Exogenous $\mathrm{H}_{2} \mathrm{~S}$ downregulates Bim expression through the PI3K/Akt-dependent signaling pathway. A significant downregulation of Bim protein was observed in the NaHS + DOX group compared with the DOX-treated group. Furthermore, co-treatment with LY294002 increased Bim protein expression compared with the control group (Fig. 7). These results indicated that pre-treatment with NaHS downregulated the expression of Bim through a PI3K/Akt-dependent signaling pathway.

Exogenous $\mathrm{H}_{2} \mathrm{~S}$ inhibits DOX-induced cytotoxicity. Fig. 8A shows that the exposure of the H9c2 cells to DOX for $24 \mathrm{~h}$ induced marked cytotoxicity, leading to a decrease in cell viability. However, cell pre-treatment with $100 \mu \mathrm{M}$ NaHS for 30 min prior to DOX exposure significantly attenuated the degree of DOX-induced cytotoxicity, as demonstrated by an increase in cell viability. The preceding results (Figs. 3 and 5) showed that $\mathrm{H}_{2} \mathrm{~S}$ attenuated the DOX-induced decrease in p-Akt in the H9c2 cells. Thus, we aimed to confirm whether the PI3K/Akt signaling pathway is involved in the protective effect of $\mathrm{H}_{2} \mathrm{~S}$. The treatment of the H9c2 cells with LY294002 and NaHS for 30 min prior to DOX exposure for $24 \mathrm{~h}$ abolished the protective effect of $\mathrm{H}_{2} \mathrm{~S}$, leading to an decrease in cell viability (Fig. 8A). NaHS alone did not alter the viability of the $\mathrm{H} 9 \mathrm{c} 2$ cells. These findings suggested that $\mathrm{H}_{2} \mathrm{~S}$ exerts a protective effect against DOX-induced cytotoxicity, which may occur through the PI3K/Akt signaling pathway.

Exogenous $\mathrm{H}_{2} \mathrm{~S}$ reduces DOX-induced apoptosis in $\mathrm{H} 9 \mathrm{c} 2$ cells. The effects of $\mathrm{H}_{2} \mathrm{~S}$ on DOX-induced apoptosis were observed. Fig. 8B shows that the apoptotic rate of the H9c2 cells exposed to $5 \mu \mathrm{M}$ of DOX for $24 \mathrm{~h}$ increased significantly. However, cell pre-treatment with $100 \mu \mathrm{M}$ NaHS for $30 \mathrm{~min}$ prior to DOX exposure markedly decreased the DOX-induced increase in the apoptotic rate. To ascertain whether the PI3K/ Akt signaling pathway is involved in apoptosis induced by DOX, the H9c2 cells were treated with LY294002 prior to exposure to NaHS plus DOX. The results revealed that pretreatment with LY294002 abolished the protective effect of $\mathrm{H}_{2} \mathrm{~S}$. NaHS alone did not markedly alter the percentage of apoptotic $\mathrm{H} 9 \mathrm{c} 2$ cells. These data strongly suggest that $\mathrm{H}_{2} \mathrm{~S}$ protects DOX-exposed H9c2 cells against apoptosis and this may occur through the PI3K/Akt signaling pathway.

Exogenous $\mathrm{H}_{2} \mathrm{~S}$ reduces DOX-induced oxidative stress in $H 9 c 2$ cells. The effect of $\mathrm{H}_{2} \mathrm{~S}$ on the DOX-induced production of ROS was investigated to elucidate whether the antioxidant activity of $\mathrm{H}_{2} \mathrm{~S}$ affords a cytoprotective effect against DOX-induced cardiotoxicity. Fig. 9 shows that the exposure of $\mathrm{H} 9 \mathrm{c} 2$ cells to $5 \mu \mathrm{M}$ DOX evidently enhanced the generation of intracellular ROS. However, NaHS pre-conditioning for 30 min markedly attenuated the DOX-elicited generation of ROS. Notably, pre-treatment with LY294002 abolished the protective effect of $\mathrm{H}_{2} \mathrm{~S}$. However, NaHS alone did not alter the basal levels of intracellular ROS.

\section{Discussion}

DOX is one of the most widely used and successful antitumor drugs, although the clinical use of DOX is limited by cumulative and dose-dependent cardiotoxic effects. With an increasing population of cancer survivors, there is a growing need to develop preventive strategies and effective therapies against DOX-induced cardiotoxicity, and particularly, late-onset cardiomyopathy. Although the cardiotoxic effects of DOX have been 


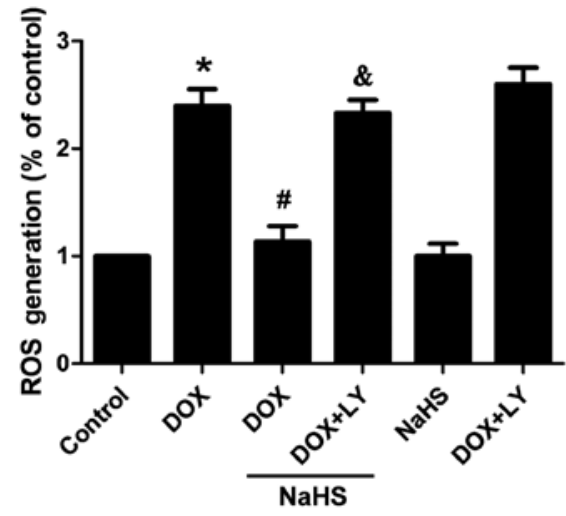

Figure 9. Exogenous hydrogen sulfide $\left(\mathrm{H}_{2} \mathrm{~S}\right)$ reduces doxorubicin (DOX)-induced ROS production in H9c2 cells. Intracellular ROS levels were determined by measuring the fluorescent intensity of DCF oxidized from DCFH-DA using a fluorescence microplate reader. Data are shown as the means \pm SEM $(n=5)$. ${ }^{*} \mathrm{P}<0.05$, compared with the control group; ${ }^{*} \mathrm{P}<0.05$, compared with the DOX-treated group; ${ }^{\text {\& }} \mathrm{P}<0.05$, compared with the sodium hydrosulfide (NaHS) + DOX group. LY, LY294002.

previously examined, the underlying mechanisms responsible for these effects remain to be elucidated. Mounting evidence supports the hypothesis that free radical-induced oxidative stress, which leads to cardiomyocyte death by apoptosis and necrosis, is a key contributor to DOX-induced cardiotoxicity (16).

Previous findings have demonstrated that exogenous $\mathrm{H}_{2} \mathrm{~S}$ offers protection against DOX-induced cardiotoxicity through antoixidant effects and the downregulation of inflammatory responses $(15,17,18)$. Guo et al have demonstrated that exogenous $\mathrm{H}_{2} \mathrm{~S}$ attenuates DOX-induced inflammation and cytotoxicity through the inhibition of the p38 MAPK/NF- $\mathrm{BB}$ pathway in $\mathrm{H} 9 \mathrm{c} 2$ cells (15). $\mathrm{H}_{2} \mathrm{~S}$ also attenuates DOX-induced cardiotoxicity through the inhibition of endoplasmic reticulum stress in $\mathrm{H} 9 \mathrm{c} 2$ cells (17). Su et al have demonstrated that the downregulation of endogenously generated $\mathrm{H}_{2} \mathrm{~S}$ is probably involved in the pathogenesis of DOX-induced cardiomyopathy, as $\mathrm{H}_{2} \mathrm{~S}$ reduces lipid peroxidation, increases the activity of antioxidant enzyme systems and inhibits oxidative stress-induced injury (18).

Erythropoietin has been found to protect the myocardium against DOX-induced impairment of heart function and inhibits the apoptosis of cardiomyocytes by activating the PI3K/Akt cell-survival pathway (19). In addition, neuregulin-1 (20), tanshinone IIA (21), and urotensin II (22) have been demonstrated to prevent the apoptosis of cardiomyocytes exposed to DOX, partly through the Akt signaling pathways. Thus, these agents may promote cell survival and exert cardioprotective effects. FoxO3a is regulated by the PI3K/Akt pathway and plays an important role in mediating the cytotoxic effects of DOX (23). It has also been demonstrated that in the presence of serum and growth factors, the survival kinase Akt is phosphorylated, which in turn phosphorylates FoxO transcription factors, thereby leading to nuclear exclusion, cytoplasmic retention and the inactivation of FoxO transcription factors (24). Conversely, oxidative stress has been shown to induce the re-localization of FoxO transcription factors from the cytoplasm to the nucleus and activate the target genes of FoxO transcription factors, including the pro-apoptotic gene Bim, with subsequent cell apoptosis (25).
The roles of FoxO3a in oxidative stress-induced cardiotoxicity have received attention. Cardiac microvascular endothelial cells (CMECs) are some of the predominant cells that are immediately damaged after myocardial I/R injury. High glucose (26) and hypoxia (27) have been observed to reduce the phosphorylation of Akt and FoxO3a, induce FoxO3a activation, and lead to ROS production and apoptosis in CMECs. Wang et al reported that venlafaxine protects PC12 cells against corticosterone-induced cell death by modulating the activity of the PI3K/Akt/FoxO3a pathway (7). Simvastatin inhibits rapamycin-induced dysfunction and apoptosis of CMECs, probably through the activation of the PI3K/ Akt/FoxO3a signaling pathway (28). Furthermore, erythropoietin activates the PI3K/Akt/FoxO3a signaling pathway and protects neurons from 6-hydroxydopamine (6-OHDA)induced apoptosis (29). In addition, sodium tanshinone IIA sulfonate (13) and bromelain (14) have been demonstrated to inhibit the FoxO3a pathway and apoptosis of cardiomyocytes. In the present study, we have demonstrated that a statistically significant reduction in the phosphorylation of Akt and FoxO3a protein was observed in the DOX-treated H9c2 cells. The data suggest that the PI3K/Akt/FoxO3a signaling pathway is important in DOX-induced cytoxicity in cardiomyocytes.

To elucidate the potential protective effects of $\mathrm{H}_{2} \mathrm{~S}$ against DOX-induced cardiotoxicity as well as the mechanisms responsible for these effects, we observed the effect of NaHS on the phosphorylation of Akt and FoxO3a protein induced by DOX exposure. The findings of the present study show that the treatment of $\mathrm{H} 9 \mathrm{c} 2$ cells with NaHS significantly prevented the DOX-induced reduction in the levels of $\mathrm{p}$-Akt and p-FoxO3a, and this was accompanied by an increase in cell viability, indicating that the $\mathrm{PI} 3 \mathrm{~K} / \mathrm{Akt} / \mathrm{FoxO} 3$ a pathway may be involved in the protective effects of exogenous $\mathrm{H}_{2} \mathrm{~S}$ against DOX-induced cardiotoxicity.

Notably, the results of the present study provide novel evidence that an interaction between ROS and FoxO3a exists in DOX-exposed $\mathrm{H} 9 \mathrm{c} 2$ cells, because $\mathrm{H}_{2} \mathrm{~S}$ attenuated the DOX-induced reduction in p-Akt and p-FoxO3a levels. Similar to exogenous $\mathrm{H}_{2} \mathrm{~S}$, the treatment of $\mathrm{H} 9 \mathrm{c} 2$ cells with NAC (ROS scavenger) prior to DOX exposure attenuated the phosphorylation of Akt and FoxO3a. These results indicated that targeting the interaction that occurs between ROS and FoxO3a in DOX-induced cardiotoxicity may aid in the treatment and prevention of cardiac injury. Another important novel finding of this study was that exogenous $\mathrm{H}_{2} \mathrm{~S}$ protects against DOX-induced cardiotoxicity by activating the PI3K/Akt/FoxO3a pathway in $\mathrm{H} 9 \mathrm{c} 2$ cells. The findings of the present study support this hypothesis. The treatment of H9c2 cells with NaHS (a donor of $\mathrm{H}_{2} \mathrm{~S}$ ) prior to DOX exposure significantly ameliorated the reduction in p-Akt and p-FoxO3a, attenuated the nuclear localization of FoxO3a and the DOX-induced apoptosis of H9c2 cells, and exerted an inhibitory effect on Bim expression. In addition, pre-treatment with LY294002, a selective inhibitor of PI3K/Akt, reversed the protective effect of $\mathrm{H}_{2} \mathrm{~S}$ on DOX-induced cardiotoxicity, as demonstrated by an increase in the number of apoptotic cells, a reduction in cell viability and the phosphorylation of Akt and FoxO3a, as well as a simultaneous increase in Bim expression. Therefore, our results suggest that the protective effects of $\mathrm{H}_{2} \mathrm{~S}$ on FoxO3a nuclear translocation and Bim expression are mediated by the PI3K/Akt pathway. 
In conclusion, to the best of our knowledge, the present study has demonstrated for the first time, that FoxO3a plays a central role in the DOX-induced apoptosis of $\mathrm{H} 9 \mathrm{c} 2$ cells. Furthermore, data from the present study have revealed that $\mathrm{H}_{2} \mathrm{~S}$ protects $\mathrm{H} 9 \mathrm{c} 2$ cardiomyocytes against DOX-induced cytotoxicity through the activation of the PI3K/Akt/FoxO3a pathway. To the best of our knowledge, this is the first study to show that $\mathrm{H}_{2} \mathrm{~S}$ is capable of acting on the PI3K/Akt/FoxO3a pathway to enhance the survival of cardiomyocytes, thereby suggesting that the FoxO3a pathway may be a novel therapeutic target in cardiovascular disease.

\section{Acknowledgements}

The present study was supported by grants from the National Natural Science Foundation of China (81470435, Z.-S.J.) and the Graduate student research innovation project of Hunan province (CX2013B397, M.-H.L.).

\section{References}

1. Magnano LC, Martínez Cibrian N, Andrade González X and Bosch X: Cardiac complications of chemotherapy: role of prevention. Curr Treat Options Cardiovasc Med 16: 312, 2014.

2. Truong J, Yan AT, Cramarossa G and Chan KK: Chemotherapy-induced cardiotoxicity: detection, prevention, and management. Can J Cardiol 30: 869-878, 2014.

3. Spagnuolo RD, Recalcati S, Tacchini L and Cairo G: Role of hypoxia-inducible factors in the dexrazoxane-mediated protection of cardiomyocytes from doxorubicin-induced toxicity. Br J Pharmacol 163: 299-312, 2011.

4. Zhang Y, Tang ZH, Ren Z, Qu SL, Liu MH, Liu LS and Jiang ZS: Hydrogen sulfide, the next potent preventive and therapeutic agent in aging and age-associated diseases. Mol Cell Biol 33: 1104-1113, 2013.

5. Osipov RM, Robich MP, Feng J, Liu Y, Clements RT, Glazer HP, Sodha NR, Szabo C, Bianchi C and Sellke FW: Effect of hydrogen sulfide in a porcine model of myocardial ischemia-reperfusion: comparison of different administration regimens and characterization of the cellular mechanisms of protection. J Cardiovasc Pharmacol 54: 287-297, 2009.

6. Huang YE, Tang ZH, Xie W, Shen XT, Liu MH, Peng XP, Zhao ZZ, Nie DB, Liu LS and Jiang ZS: Endogenous hydrogen sulfide mediates the cardioprotection induced by ischemic postconditioning in the early reperfusion phase. Exp Ther Med 4: $1117-1123,2012$.

7. Wang H, Zhou X, Huang J, Mu N, Guo Z, Wen Q, Wang R, Chen S, Feng ZP and Zheng W: The role of Akt/FoxO3a in the protective effect of venlafaxine against corticosterone-induced cell death in PC12 cells. Psychopharmacology (Berl) 228: 129-141, 2013.

8. van der Vos KE and Coffer PJ: The extending network of FOXO transcriptional target genes. Antioxid Redox Signal 14: 579-592, 2011.

9. Sanphui $\mathrm{P}$ and Biswas SC: FoxO3a is activated and executes neuron death via Bim in response to $\beta$-amyloid. Cell Death Dis 4: e625, 2013

10. Bao W, Pan F, Chen L, Su G, Gao X, Li Y, Sun Q, Sun J, He K and Song H: The PI3K/AKT pathway and FOXO3a transcription factor mediate high glucose-induced apoptosis in neonatal rat ventricular myocytes. Iran Red Crescent Med J 16: e14914, 2014

11. Li D, Qu Y, Mao M, Zhang X, Li J, Ferriero D and Mu D: Involvement of the PTEN-AKT-FOXO3a pathway in neuronal apoptosis in developing rat brain after hypoxia-ischemia. J Cereb Blood Flow Metab 29: 1903-1913, 2009.

12. Liu MH, Yuan C, He J, Tan TP, Wu SJ, Fu HY, Liu J, Yu S, Chen YD, Le QF, et al: Resveratrol protects PC12 cells from high glucose-induced neurotoxicity via PI3K/Akt/FoxO3a pathway. Cell Mol Neurobiol 35: 513-522, 2014.
13. Zhang MQ, Zheng YL, Chen H, Tu JF, Shen Y, Guo JP, Yang XH, Yuan SR, Chen LZ, Chai JJ, et al: Sodium tanshinone IIA sulfonate protects rat myocardium against ischemia-reperfusion injury via activation of $\mathrm{PI} 3 \mathrm{~K} / \mathrm{Akt} / \mathrm{FOXO} \mathrm{A} / \mathrm{Bim}$ pathway. Acta Pharmacol Sin 34: 1386-1396, 2013.

14. Juhasz B, Thirunavukkarasu M, Pant R, Zhan L, Penumathsa SV, Secor ER Jr, Srivastava S, Raychaudhuri U, Menon VP, Otani H, et al: Bromelain induces cardioprotection against ischemia-reperfusion injury through $\mathrm{Akt} / \mathrm{FOXO}$ pathway in rat myocardium. Am J Physiol Heart Circ Physiol 294: H1365-H1370, 2008.

15. Guo R, Wu K, Chen J, Mo L, Hua X, Zheng D, Chen P, Chen G, $\mathrm{Xu} \mathrm{W}$ and Feng J: Exogenous hydrogen sulfide protects against doxorubicin-induced inflammation and cytotoxicity by inhibiting p38MAPK/NFkB pathway in H9c2 cardiac cells. Cell Physiol Biochem 32: 1668-1680, 2013.

16. Tocchetti CG, Carpi A, Coppola C, Quintavalle C, Rea D, Campesan M, Arcari A, Piscopo G, Cipresso C, Monti MG, et al: Ranolazine protects from doxorubicin-induced oxidative stress and cardiac dysfunction. Eur J Heart Fail 16: 358-366, 2014.

17. Wang XY, Yang CT, Zheng DD, Mo LQ, Lan AP, Yang ZL, Hu F, Chen PX, Liao XX and Feng JQ: Hydrogen sulfide protects H9c2 cells against doxorubicin-induced cardiotoxicity through inhibition of endoplasmic reticulum stress. Mol Cell Biochem 363: 419-426, 2012.

18. Su YW, Liang C, Jin HF, Tang XY, Han W, Chai LJ, Zhang CY, Geng B, Tang CS and Du JB: Hydrogen sulfide regulates cardiac function and structure in adriamycin-induced cardiomyopathy. Circ J 73: 741-749, 2009.

19. Kim KH, Oudit GY and Backx PH: Erythropoietin protects against doxorubicin-induced cardiomyopathy via a phosphatidylinositol 3-kinase-dependent pathway. J Pharmacol Exp Ther 324: 160-169, 2008.

20. An T, Zhang Y, Huang Y, Zhang R, Yin S, Guo X, Wang Y, Zou C, Wei B, Lv R, et al: Neuregulin-1 protects against doxorubicin-induced apoptosis in cardiomyocytes through an Akt-dependent pathway. Physiol Res 62: 379-385, 2013.

21. Hong HJ, Liu JC, Chen PY, Chen JJ, Chan P and Cheng TH: Tanshinone IIA prevents doxorubicin-induced cardiomyocyte apoptosis through Akt-dependent pathway. Int J Cardiol 157: 174-179, 2012.

22. Chen YL, Loh SH, Chen JJ and Tsai CS: Urotensin II prevents cardiomyocyte apoptosis induced by doxorubicin via Akt and ERK. Eur J Pharmacol 680: 88-94, 2012.

23. Ho KK, McGuire VA, Koo CY, Muir KW, de Olano N, Maifoshie E, Kelly DJ, McGovern UB, Monteiro LJ, Gomes AR, et al: Phosphorylation of FOXO3a on Ser-7 by p38 promotes its nuclear localization in response to doxorubicin. $\mathrm{J}$ Biol Chem 287: 1545-1555, 2012.

24. Wang Y, Zhou Y and Graves DT: FOXO transcription factors: their clinical significance and regulation. Biomed Res Int 2014: 925350, 2014

25. Storz P: Forkhead homeobox type O transcription factors in the responses to oxidative stress. Antioxid Redox Signal 14: 593-605, 2011.

26. Peng C, Ma J, Gao X, Tian P, Li W and Zhang L: High glucose induced oxidative stress and apoptosis in cardiac microvascular endothelial cells are regulated by FoxO3a. PLoS One 8: e79739, 2013.

27. Zhang S, Zhao Y, Xu M, Yu L, Zhao Y, Chen J, Yuan Y, Zheng Q and Niu X: FoxO3a modulates hypoxia stress induced oxidative stress and apoptosis in cardiac microvascular endothelial cells. PLoS One 8: e80342, 2013.

28. Pan Q, Xie X, Guo Y and Wang H: Simvastatin promotes cardiac microvascular endothelial cells proliferation, migration and survival by phosphorylation of p70 S6K and FoxO3a. Cell Biol Int 38: 599-609, 2014.

29. Jia Y, Mo SJ, Feng QQ, Zhan ML, OuYang LS, Chen JC, Ma YX, Wu JJ and Lei WL: EPO-dependent activation of $\mathrm{PI} 3 \mathrm{~K} / \mathrm{Akt} / \mathrm{FoxO}$ a signalling mediates neuroprotection in in vitro and in vivo models of Parkinson's disease. J Mol Neurosci 53: 117-124, 2014. 